\title{
SGLT2-Hemmer abgewatscht
}

Das Institut für Qualität und Wirtschaftlichkeit im Gesundheitswesen (IQWiG) äußert Zweifel an der internationalen Studie zum kardiovaskulären Outcome bei Typ-2-Diabetikern mit EmpagliflozinTherapie. Die Begründungen lassen staunen.

Einmal mehr erkennt das IQWiG bei einer SGLT2-HemmerTherapie von Patienten mit Typ-2-Diabetes keinen Zusatznutzen. Am Mittwoch hat das Institut die Ergebnisse der frühen Nutzenbewertung von Empagliflozin vorgelegt. Es handelt sich um eine Neuauflage einer Bewertung aus dem Jahr 2014, die nötig wurde, weil der Hersteller weitere Studiendaten eingereicht hatte. Dabei geht es zum einen um die antidiabetische Wirkung bzw. Nebenwirkungsprofile. Hier sieht das IQWiG auch nach Durchsicht der neuen Daten keinen Anhalt für einen Zusatznutzen gegenüber der zweckmäßigen Vergleichstherapie. Die war, in Analogie zu anderen Nutzenbewertungen beim Typ2-Diabetes, definiert als „Metformin plus Sulfonylharnstoff“ oder "Metformin plus Insulin“ oder, wenn Metformin nicht vertragen wird, Sulfonylharnstoff alleine. Was diesen „diabetischen" Part der Bewertung angeht, macht das IQWiG wie im Jahr 2014 geltend, dass die Daten, insbesondere zu spezifischen unerwünschten Ereignissen, bei denen sich ein Nachteil von Empagliflozin zeigte, unvollständig seien. Auch seien die Studienergebnisse teilweise widersprüchlich.

\section{Kardiovaskuläre Mortalität signifikant verringert}

Daneben äußert sich das IQWiG auch zur EMPA-REG OUTCOME-Studie, einer placebokontrollierten Großstudie mit kardiovaskulärem Endpunkt. Zur Erinnerung: 7.028 Typ-2-Diabetiker mit hohem kardiovaskulärem Risiko wurden entweder wie bisher mit oralen Antidiabetika behandelt, oder sie erhielten zusätzlich Empagliflozin. Nach im Mittel 3,1 Jahren hatten in der Empagliflozin-Gruppe 10,5\% der Patienten ein primäres Endpunktereignis (kardiovaskulärer Tod oder nicht-tödlicher Myokardinfarkt/Schlaganfall). In der Vergleichsgruppe waren es $12,1 \%$, eine relative Risikoreduktion um $14 \%$ und eine NumberNeeded-to-Treat von 62,5 ( $\mathrm{p}=0,04)$. Getrieben wurde der Vorteil fast ausschließlich durch die Reduktion der kardiovaskulären Sterblichkeit (NEJM 2015;373:2117).

\section{Fachgesellschaften äußern sich positiv}

Die Studie hat für Aufsehen gesorgt, weil es die erste randomisierte Studie war, bei der ein orales Antidiabetikum das kardiovaskuläre Outcome verbessert hat. Für andere SGLT2-Hemmer konnte das bisher nicht gezeigt werden. Trotzdem wird die Studie von Diabetologen und Kardiologen vorsichtig positiv bewertet. So hält die Deutsche Diabetes-Gesellschaft (DDG) die Studie für „überzeugend“, insbesondere vor dem Hintergrund einer schon optimalen Lipid- und Blutdrucksenkung. Als Mechanismus wird eine osmotisch-diuretische Wirkung diskutiert, also kein antiatherosklerotischer Effekt, sondern ein günstiger Effekt auf Herzfunktion/Herzinsuffizienz. Entsprechend er- wähnt auch die Europäische Gesellschaft für Kardiologie die EMPA-REG OUTCOME-Studie in ihrer neuen HerzinsuffizienzLeitlinie mehrfach, weist aber auch darauf hin, dass es eine Einzelstudie ist. Was sagt nun das IQWiG zur EMPA-REG OUTCOME-Studie? Kurz gesagt: Sie fällt komplett durch, wird als „vertane Chance“ bezeichnet, mit der der Hersteller eine „selbstgestellte Frage" beantworten möchte. Weder würden die Fragestellungen des GBA adressiert noch dessen zweckmäßige Vergleichstherapien genutzt. Auch wird kritisiert, dass in der Studie die antidiabetische Therapie nicht in „erforderlicher Weise" eskaliert worden sei und dass die Studienärzte die ,in den Leitlinien genannten oberen Grenzwerte" nicht konsequent beachtet hätten.

\section{Angreifbare Kritikpunkte}

Das sind starke Worte. EMPA-REG OUTCOME war eine der kardiovaskulären Sicherheitsstudien, die die FDA seit 2008 als Konsequenz aus den Glitazon-Studien standardmäßig von neuen oralen Antidiabetika verlangt. Zumindest in diesem Punkt war es also keine selbstgestellte Frage. Auch die Protokollvorgabe, wonach in den ersten zwölf Wochen die Hintergrundtherapie nur in Notfällen eskaliert werden durfte, hängt damit zusammen. Bei den Vergleichstherapien gilt, was in ähnlichen Fällen immer wieder betont wird: GBA und IQWiG sind nicht zwangsläufig die ersten Anlaufstellen, wenn es bei einer in 42 Ländern durchgeführten Studie mit über 7.000 Teilnehmern um die Festlegung des Studiendesigns geht. Und der wiederholte Verweis auf Forderungen einer Nutzenbewertung aus dem Jahr 2014 überzeugt bei einer Studie, die 2010 startete, auch nicht wirklich.

\section{IQWiG ohrfeigt EMPA-REG OUTCOME-Studie}

Merkwürdig ist auch der Hinweis, wonach die Vorteile von Empagliflozin vor allem in Lateinamerika und Asien aufgetreten seien. Zum einen werden damit geografische Regionen in einer Weise in den Vordergrund gerückt, die bei anderen nicht-prädefinierten Subgruppen niemals akzeptiert würde. Zum anderen sind die Daten auch durchaus vielgestaltig. Zwar lässt sich im Supplementary Index der Studie beim primären Endpunkt in Europa tatsächlich kein Vorteil ausmachen. Bei dem für das Gesamtergebnis relevanten Endpunkt „kardiovaskulärer Tod“ schneidet Europa aber ähnlich ab wie Asien und Lateinamerika. Auch was Ethnien bzw. Rassen angeht, sind die Daten keineswegs extrem heterogen: Kardiovaskuläre Todesereignisse waren unter Empagliflozin nicht nur bei Latinos und Asiaten seltener, sondern auch bei Weißen. Ganz so einfach scheint es also nicht zu sein. Das alles heißt nicht, dass zur Frage der kardiovaskulären Folgen einer Empagliflozin-Therapie nicht weitere Daten nötig sind. Das sind sie sicher.

Die EMPA-REG OUTCOME-Studie ist auch nicht über Kritik erhaben. Ob sich die deutsche Nutzenbewertungsbürokratie mit einer derartigen Ohrfeige und vor allem mit diesen Begründungen aber einen Gefallen getan hat, ist die andere Frage.

Philipp Grätzel von Grätz

Erschienen am 02.06.2016 in der Ärzte Zeitung 